\title{
TAIL ASYMPTOTICS OF LIGHT-TAILED WEIBULL-LIKE SUMS
}

\author{
BY
}

\author{
SØREN ASMUSSEN (AARHUS), ENKELEJD HASHORVA (LAUSANNE), \\ PATRICK J. LAUB (BRISBANE), AND THOMAS TAIMRE (BRISBANE)
}

This paper is dedicated to Tomasz Rolski in appreciation of his fundamental contributions to applied probability over a lifetime, and of his sustaining friendship.

\begin{abstract}
We consider sums of $n$ i.i.d. random variables with tails close to $\exp \left\{-x^{\beta}\right\}$ for some $\beta>1$. Asymptotics developed by Rootzén (1987) and Balkema, Klüppelberg, and Resnick (1993) are discussed from the point of view of tails rather than of densities, using a somewhat different angle, and supplemented with bounds, results on a random number $N$ of terms, and simulation algorithms.
\end{abstract}

2010 AMS Mathematics Subject Classification: Primary: 62E20, 60F05; Secondary: 60E15, 65C05, 60F10.

Key words and phrases: Gumbel MDA, Laplace transform, rareevent simulation, sums of of random variables, Weibull distribution.

\section{INTRODUCTION}

Let $X, X_{1}, \ldots, X_{n}$ be i.i.d. with common distribution $F$. A recurrent theme in applied probability is then to determine the order of magnitude of the tail $\mathbb{P}\left(S_{n}>x\right)$ of their sum $S_{n}=X_{1}+\ldots+X_{n}$.

The results vary according to the heaviness of the tail $\bar{F}=1-F$ of $F$. In the heavy-tailed case, defined as the $X$ for which $\mathbb{E} \mathrm{e}^{s X}=\infty$ for all $s>0$, there is a subexponential class in which the results take a clean form (see, e.g., [12] or [3]). In fact, by the very definition of subexponentiality, we have $\mathbb{P}\left(S_{n}>x\right) \sim n \bar{F}(x)$ as $x \rightarrow \infty$, where $\bar{F}(x)=\mathbb{P}(X>x)$. The main examples are regularly varying $\bar{F}(x)$, lognormal $X$, and Weibull tails $\bar{F}(x)=\mathrm{e}^{-c x^{\beta}}$, where $0<\beta<1$.

In the light-tailed case, defined as the $X$ for which $\mathbb{E}^{s X}<\infty$ for some $s>0$, the most standard asymptotic regime is not $x \rightarrow \infty$ but rather $x=x_{n}$ going to infinity at rate $n$. For example, let $x_{n}=n z$ for some $z$, where typically $z>\mathbb{E} X$ in order to make the problem a rare-event one. Under some regularity conditions, 
the sharp asymptotics are then given by the saddlepoint approximation $\mathbb{P}\left(S_{n}>x\right)$ $\sim c(z) \mathrm{e}^{-n I(z)} / n^{1 / 2}$ for suitable $c(z)$ and $I(z)$, cf. [114]. This is a large deviations result, describing how likely it is for $S_{n}$ to be far from the value $n \mathbb{E} X$ predicted by the LLN. However, in many applications the focus is rather on a small or moderate $n$, i.e. the study of $\mathbb{P}\left(S_{n}>x\right)$ as $x \rightarrow \infty$ with $n$ fixed.

The basic light-tailed explicit examples in this setting are the exponential distribution, the gamma distribution, the inverse Gaussian distribution, and the normal distribution. The tail of $F$ is exponential or close-to-exponential for exponential, gamma and inverse Gaussian distributions; this is the borderline between light and heavy tails, and the analysis of tail behaviour is relatively simple in this case (we give a short summary later in Section 8 ). The most standard class of distributions with a lighter tail is formed by the Weibull distributions where $\bar{F}(x)=\mathrm{e}^{-c x^{\beta}}$ for some $\beta>1$. For $\beta=2$, this is close to the normal distribution, where (by its well-known Mill's ratio) $\bar{F}(x) \sim \mathrm{e}^{-x^{2} / 2} /(\sqrt{2 \pi} x)$ when $F=\Phi$ is the standard normal law. The earliest study of tail properties of $S_{n}$ may be that of [16] which was later followed up by the mathematically deeper and somewhat general study of Balkema, Klüppelberg, and Resnick [7], henceforth referred to as BKR. The setting of both papers concerns densities.

Despite filling an obvious place in the theory of tails of sums, it has been our impression that this theory is less known than it should be. This was confirmed by a Google Scholar search which gave only 27 citations of BKR, most of which were even rather peripheral. One reason may be that the title Densities with Gaussian tails of BKR is easily misinterpreted; another, the heavy analytic flavour of the paper. Also note that the focus of [16] is somewhat different, and the set of results we are interested in here appears as a by-product at the end of that paper.

The purpose of the present paper is twofold: to present a survey from a somewhat different angle than BKR, in the hope of somewhat remedying this situation; and to supplement the theory with various new results. In the survey part, the aim has been simplicity and intuition more than generality. In particular, we avoid considering convex conjugates and some non-standard central limit theory developed in Section 6 of BKR. These tools are mathematically deep and elegant, but not really indispensable for developing what we see as the main part of the theory. Beyond this expository aspect, our contributions are: to present the main results and their conditions in terms of tails rather than densities; to develop simple upper and lower bounds; to study the case of a random number of terms $N$, more precisely properties of $\mathbb{P}\left(S_{N}>x\right)$ when $N$ is an independent Poisson r.v.; and to look into simulation aspects.

The precise assumptions on the distribution $F$ in the paper vary somewhat depending on the context and progression of the paper. The range goes from the vanilla Weibull tail $\bar{F}(x)=\mathrm{e}^{-c x^{\beta}}$ via an added power in the asymptotics, $\bar{F}(x) \sim$ $d x^{\alpha} \mathrm{e}^{-c x^{\beta}}$, to the full generality of the BKR set-up. Here $c x^{\beta}$ is replaced by a smooth convex function $\psi(x)$ satisfying $\psi^{\prime}(x) \rightarrow \infty$, and the density has the form 
$\gamma(x) \mathrm{e}^{-\psi(x)}$ for a function $\gamma$ which is in some sense much less variable than $\psi$ (the precise regularity conditions are given in Section 4 ).

\section{HEURISTICS}

With heavy tails, the basic intuition on the tail behaviour of $S_{n}$ is the principle of a single big jump; this states that a large value of $S_{n}$ is typically caused by one summand being large while the rest take ordinary values. A rigorous formulation of this can be proved in a few lines from the very definition of subexponentiality, see, e.g., [3], p. 294. With light tails, the folklore is that if $S_{n}$ is large, say $S_{n} \approx x$, then all $X_{i}$ are of the same order $x / n$.

This suggest that the asymptotics of $\mathbb{P}\left(S_{n}>x\right)$ are essentially determined by the form of $F$ locally around $x / n$. A common type of such local behaviour is that $\bar{F}(x+e(x) y) \sim \bar{F}(x) \mathrm{e}^{-y}$ for some positive function $e(x)$ as $x \rightarrow \infty$ with $y \in \mathbb{R}$ fixed; this is abbreviated as $F \in \operatorname{GMDA}(e)$. Equivalently,

$$
\Lambda(x+e(x) y) \sim \Lambda(x)+y,
$$

where $\Lambda(x)=-\log \bar{F}(x)$. Here one can take $e(x)=\mathbb{E}[X-x \mid X>x]$, the socalled mean excess function; if $F$ admits a density $f(x)$, an alternative asymptotically equivalent choice is the inverse hazard rate $e(x)=1 / \lambda(x)$, where $\lambda(x)=$ $\Lambda^{\prime}(x)=f(x) / \bar{F}(x)$.

In fact, (2. I) is a necessary and sufficient condition for $F$ to be in $\operatorname{GMDA}(e)$, the maximum domain of attraction of the Gumbel distribution [12]. Even if this condition may look special at first sight, it covers the vast majority of well-behaved light-tailed distributions, with some exceptions such as certain discrete distributions like the geometric or Poisson.

From these remarks one may proceed for $n=2$ from the convolution,

$$
\begin{aligned}
& \text { (2.2) } \mathbb{P}\left(X_{1}+X_{2}>x\right)=(f * \bar{F})(x)=\int_{-\infty}^{\infty} \lambda(z) \exp \{-\Lambda(z)-\Lambda(x-z)\} \mathrm{d} z \\
& =\int_{-\infty}^{\infty} \frac{e(x / 2)}{e(x / 2+e(x / 2) y)} \exp \left\{-\Lambda\left(\frac{x}{2}+e\left(\frac{x}{2}\right) y\right)-\Lambda\left(\frac{x}{2}-e\left(\frac{x}{2}\right) y\right)\right\} \mathrm{d} y,
\end{aligned}
$$

where we have substituted $z=x / 2+e(x / 2) y$. First, note that if $\lambda(x)$ tends to zero as $x \rightarrow \infty$ and is differentiable, we can expand $\Lambda$ about $y=0$ as

$$
\Lambda\left(\frac{x}{2}+e\left(\frac{x}{2}\right) y\right) \sim \Lambda\left(\frac{x}{2}\right)+y+\frac{\lambda^{\prime}(x / 2)}{2 \lambda(x / 2)^{2}} y^{2} .
$$

By defining $\sigma^{2}(u)=\lambda(u)^{2} / 2 \lambda^{\prime}(u)$ and repeating this argument, we get

$$
\Lambda\left(\frac{x}{2} \pm e\left(\frac{x}{2}\right) y\right) \sim \Lambda\left(\frac{x}{2}\right) \pm y+\frac{y^{2}}{4 \sigma^{2}(x / 2)} .
$$


Also, we will use that $e(x)$ is self-neglecting, i.e. for all $t, e(x+e(x) t) \sim e(x)$ as $x \rightarrow \infty$, as is well known and easy to prove from (2.II). Combining (2.3) and the self-neglecting property with (2.2) gives us

$$
\begin{aligned}
\mathbb{P}\left(X_{1}+X_{2}>x\right) & \sim \int_{-\infty}^{\infty} 1 \cdot \exp \left\{-2 \Lambda\left(\frac{x}{2}\right)-\frac{y^{2}}{2 \sigma^{2}(x / 2)}\right\} \mathrm{d} y \\
& =\sqrt{2 \pi \sigma^{2}(x / 2)} \exp \{-2 \Lambda(x / 2)\} .
\end{aligned}
$$

In summary, rewriting (2.4) gives

$$
\overline{F^{* 2}}(x)=\mathbb{P}\left(X_{1}+X_{2}>x\right) \sim \bar{F}(x / 2)^{2} \sqrt{\pi \frac{\lambda(x / 2)^{2}}{\lambda^{\prime}(x / 2)}} .
$$

The key issue in making this precise is to keep better track of the second order term in the Taylor expansion, as discussed later in the paper.

REMARK 2.1. The procedure to arrive at (2.5) is close to the Laplace method for obtaining integral asymptotics. Classically, the integral in question has the form $\int_{a}^{b} \mathrm{e}^{-\theta h(z)} \mathrm{d} z$, and one proceeds by finding the $z_{0}$ at which $h(z)$ is minimum and performing a second order Taylor expansion around $z_{0}$. Here, we neglected the $\lambda(z)$ in front and took the relevant analogue of $z_{0}$ as $x / 2$, which is precisely the minimizer of $\Lambda(x-z)+\Lambda(z)$.

REMARK 2.2. If $X_{1}, X_{2}$ have different distributions $F_{1}, F_{2}$, the above calculations suggest that $X_{1}+X_{2}>x$ will occur roughly when $X_{1} \approx z(x), X_{2} \approx x-$ $z(x)$, where $z=z(x)$ is the solution of $\lambda_{1}(z)=\lambda_{2}(x-z)$. In fact, this is what is needed to make the first order Taylor terms cancel. For example, if $\bar{F}_{1}(x)=\mathrm{e}^{-x^{\beta_{1}}}$, $\bar{F}_{2}(x)=\mathrm{e}^{-x^{\beta_{2}}}$ with $\beta_{2}<\beta_{1}$, we get $z(x) \sim c x^{\eta}$, where $\eta=\left(\beta_{2}-1\right) /\left(\beta_{1}-1\right)<1$, $c=\left(\beta_{2} / \beta_{1}\right)^{1 /\left(\beta_{1}-1\right)}$. This type of heuristic is an important guideline when designing importance sampling algorithms, cf. [4], V.1, VI.2.

\section{WEIBULL-LIKE SUMS}

We now make the heuristics of the preceding section rigorous for the case of different distributions $F_{1}, F_{2}$ of $X_{1}, X_{2}$ such that the densities $f_{1}, f_{2}$ satisfy

$$
f_{i}(x) \sim d_{i} x^{\alpha_{i}+\beta-1} \mathrm{e}^{-c_{i} x^{\beta}}, \quad x \rightarrow \infty, i=1,2,
$$

for some common $\beta>1$, where the $\alpha_{i}$ can take any value in $(-\infty, \infty)$, and $c_{i}, d_{i}$ are positive $(i=1,2)$.

We start by some analytic preliminaries. Given (B.]), we define

$$
\eta=c_{1}^{1 /(\beta-1)}+c_{2}^{1 /(\beta-1)}, \quad \theta_{1}=\frac{c_{2}^{1 /(\beta-1)}}{\eta}, \quad \theta_{2}=\frac{c_{1}^{1 /(\beta-1)}}{\eta}, \quad \kappa=\frac{\eta^{\beta-1}}{\beta c_{1} c_{2}} .
$$


Note that

$$
\bar{F}_{i}(x) \sim \frac{d_{i}}{\beta c_{i}} x^{\alpha_{i}} \mathrm{e}^{-c_{i} x^{\beta}}
$$

(hence $c_{i}=1, d_{i}=\beta, \alpha_{i}=0$ corresponds to the traditional Weibull tail $\mathrm{e}^{-x^{\beta}}$ ). Define the excess function of $F_{i}$ by $e_{i}(x)=\bar{F}_{i}(x) / f_{i}(x)$. Thus $e_{i}(x)$ is the inverse hazard rate and has asymptotics $x^{1-\beta} /\left(\beta c_{i}\right)$ with limit zero as $x \rightarrow \infty$. and

Lemma 3.1. Define $c=c_{1} \theta_{1}^{\beta}+c_{2} \theta_{2}^{\beta}$. Then $c<\min \left(c_{1}, c_{2}\right), \theta_{1}+\theta_{2}=1$,

$$
e_{1}\left(\theta_{1} x\right) \sim e_{2}\left(\theta_{2} x\right) \sim \frac{\kappa}{x^{\beta-1}}=\frac{1}{\beta c_{1} \theta_{1}^{\beta-1} x^{\beta-1}}=\frac{1}{\beta c_{2} \theta_{2}^{\beta-1} x^{\beta-1}} .
$$

P r o of. All statements are obvious except $c<\min \left(c_{1}, c_{2}\right)$. But

$$
\begin{aligned}
c & =c_{1} \theta_{1}^{\beta-1} \theta_{1}+c_{2} \theta_{2}^{\beta-1} \theta_{2}=\frac{c_{1} c_{2} \theta_{1}}{\eta^{\beta-1}}+\frac{c_{1} c_{2} \theta_{2}}{\eta^{\beta-1}}=\frac{c_{1} c_{2}}{\eta^{\beta-1}} \\
& <\frac{c_{1} c_{2}}{\left[c_{2}^{1 /(\beta-1)}\right]^{\beta-1}}=c_{1} .
\end{aligned}
$$

Similarly, $c<c_{2}$.

LEMMA 3.2. We have

$$
(1+h)^{\beta}=1+h \beta+\frac{h^{2}}{2} \beta(\beta-1) \omega(h),
$$

where $\omega(h) \rightarrow 1$ as $h \rightarrow 0$ and $\underline{\omega}_{\varepsilon}=\inf _{-1+\varepsilon<h<\varepsilon^{-1}} \omega(h)>0$ for all $\varepsilon>0$.

Proof. By standard Taylor expansion results, $\omega(h)=\left(1+h^{*}\right)^{\beta-2}$, where $h^{*}$ is between 0 and $h$. The statement on $\underline{\omega}_{\varepsilon}$ follows from this by considering all four combinations of the cases $h \leqslant 0$ or $h>0,1<\beta \leqslant 2$ or $\beta \geqslant 2$ separately.

The key result is the following. It allows us, for example, to determine the asymptotics of the tail or density of $F^{* n}$ in the Weibull-like class by a straightforward induction argument, see Corollary 3.1 below.

THEOREM 3.1. Under the assumption (B. I), $\mathbb{P}\left(X_{1}+X_{2}>x\right) \sim k x^{\gamma} \mathrm{e}^{-c x^{\beta}}$ as $x \rightarrow \infty$, where $\gamma=\alpha_{1}+\alpha_{2}+\beta / 2$ and $k=d_{1} d_{2} \theta_{1}^{\alpha_{1}} \theta_{2}^{\alpha_{2}} \kappa \eta^{1-\beta}\left(2 \pi \sigma^{2}\right)^{1 / 2} / \beta$, with $\theta_{1}, \theta_{2}, \kappa, \eta$ as in (B.2), the constant $c$ as in Lemma B.], and $\sigma^{2}$ determined by the equality

$$
\frac{1}{\sigma^{2}}=\frac{1}{\sigma_{1}^{2}}+\frac{1}{\sigma_{2}^{2}}, \quad \text { where } \frac{1}{\sigma_{i}^{2}}=\beta(\beta-1) c_{i} \theta_{i}^{\beta-2} \kappa^{2} .
$$

Further, the density of $X_{1}+X_{2}$ has the asymptotic form $\beta c k x^{\gamma+\beta-1} \mathrm{e}^{-c x^{\beta}}$. 
REMARK 3.1. If $F_{1}=F_{2}$ and $c_{1}=c_{2}=1$, then $\theta_{1}=\theta_{2}=1 / 2$ and $c=$ $1 / 2^{\beta-1}$ in accordance with Section 2 .

Pro of. By Lemma B.], we can choose $0<a_{-}<a_{+}<1$ such that $a_{+}^{\beta} c_{2}>c$, $\left(1-a_{-}\right) c_{1}>c$. Then

$$
\mathbb{P}\left(X_{1}+X_{2}>x, X_{1} \notin\left[a_{-} x, a_{+} x\right]\right) \leqslant \mathbb{P}\left(X_{1}>a_{+} x\right)+\mathbb{P}\left(X_{2}>\left(1-a_{-}\right) x\right)
$$

is $o\left(x^{\gamma} \mathrm{e}^{-c x^{\beta}}\right)$, and so it suffices to show that

$$
\mathbb{P}\left(X_{1}+X_{2}>x, a_{-} x<X_{1}<a_{+} x\right)=\int_{a_{-}}^{a_{+} x} f_{1}(z) \bar{F}_{2}(x-z) \mathrm{d} z
$$

has the claimed asymptotics. The last expression together with $a_{-}>0, a_{+}<1$ also shows that the asymptotics is a tail property, so that without loss of generality we may assume that $e_{i}\left(\theta_{i} x\right)=\kappa / x^{\beta-1}$, implying that (B.4) holds with equality.

Now

$$
\begin{aligned}
\mathbb{P}\left(X_{1}+\right. & \left.X_{2}>x, a_{-}<X_{1}<a_{+} x\right)=\int_{a_{-} x}^{a_{+} x} f_{1}(z) \bar{F}_{2}(x-z) \mathrm{d} z \\
& =\int_{a_{-} x}^{a_{+} x} \frac{d_{1} d_{2}}{\beta c_{2}} z^{\alpha_{1}+\beta-1}(x-z)^{\alpha_{2}} \exp \left\{-c_{1} z^{\beta}-c_{2}(x-z)^{\beta}\right\} \mathrm{d} z .
\end{aligned}
$$

Using the substitution $z=\theta_{1} x+y \kappa / x^{\beta-1}$, we have $x-z=\theta_{2} x-y \kappa / x^{\beta-1}$,

$$
c_{1} z^{\beta}+c_{2}(x-z)^{\beta}=c_{1} \theta_{1}^{\beta} x^{\beta}\left(1+h_{1}(x, y)\right)^{\beta}+c_{2} \theta_{2}^{\beta} x^{\beta}\left(1-h_{2}(x, y)\right)^{\beta},
$$

where $h_{i}(x, y)=y \kappa /\left(\theta_{i} x^{\beta}\right)$. By Taylor expanding $\left(1 \pm h_{i}(x, y)\right)^{\beta}$ as in Lemma B.2 and using (B.4), the first order term of (3.8) is

$$
c_{1} \theta_{1}^{\beta} x^{\beta}+c_{2} \theta_{2}^{\beta} x^{\beta}+\beta c_{1} \theta_{1}^{\beta-1} \kappa-\beta c_{2} \theta_{2}^{\beta-1} \kappa=c x^{\beta} .
$$

Defining $\omega_{1}(x, y)=\omega\left(h_{1}(x, y)\right), \omega_{2}(x, y)=\omega\left(-h_{2}(x, y)\right)$, we obtain the second line of (B.7) in the form

$$
\begin{aligned}
& \frac{d_{1} d_{2}}{\beta c_{2}} \int_{y_{-}(x)}^{y_{+}(x)}\left(\theta_{1} x+e_{1}\left(\theta_{1} x\right) y\right)^{\alpha_{1}+\beta-1}\left(\theta_{2} x-e_{2}\left(\theta_{2} x\right) y\right)^{\alpha_{2}} \\
& \quad \times \exp \left\{-c x^{\beta}-\frac{y^{2}}{2 \sigma_{1}^{2} x^{\beta}} \omega_{1}(x, y)-\frac{y^{2}}{2 \sigma_{2}^{2} x^{\beta}} \omega_{2}(x, y)\right\} \frac{\kappa}{x^{\beta-1}} \mathrm{~d} y
\end{aligned}
$$

where $y_{-}(x)=\left(a_{-}-\theta_{1}\right) x^{\beta} / \kappa, y_{+}(x)=\left(a_{+}-\theta_{1}\right) x / e\left(\theta_{1} x\right)$. Notice here that $a_{-} x<z<a_{+} x$ ensures the bound

$$
h_{1}(x, y)=\frac{1}{\theta_{1} x}\left(z-\theta_{1} x\right) \geqslant \frac{a_{-}}{\theta_{1}}-1>-1 .
$$


Similarly, $-h_{2}(x, y) \geqslant-a_{+} / \theta_{2}-1>1$. Using Lemmas 3.1 and 3.2 shows that the $\omega_{i}(x, y)$ are uniformly bounded below, and that $\left(\theta_{i} x+e_{i}\left(\theta_{i} x\right) y\right) / x$ is bounded in $y_{-}(x)<y<y_{+}(x)$ and goes to $\theta_{i}$ as $x \rightarrow \infty$. A dominated convergence argument proves therefore that the asymptotics of (B.6) is the same as that of

$$
\begin{aligned}
& \frac{d_{1} d_{2} \kappa}{\beta c_{2}} \theta_{1}^{\alpha_{1}+\beta-1} \theta_{2}^{\alpha_{2}} x^{\alpha_{1}+\alpha_{2}} \mathrm{e}^{-c x^{\beta}} \int_{-\infty}^{\infty} \exp \left\{-\frac{y^{2}}{2 \sigma^{2} x^{\beta}}\right\} \mathrm{d} y \\
& =\frac{d_{1} d_{2} \kappa \eta^{1-\beta}}{\beta} \theta_{1}^{\alpha_{1}} \theta_{2}^{\alpha_{2}} x^{\alpha_{1}+\alpha_{2}} \mathrm{e}^{-c x^{\beta}}\left(2 \pi \sigma^{2} x^{\beta}\right)^{1 / 2}=k x^{\gamma} \mathrm{e}^{-c x^{\beta}} .
\end{aligned}
$$

This proves the assertion on the tail of $X_{1}+X_{2}$, and the proof of the density claim differs only by constants.

Corollary 3.1. Assume the density $f$ of $F$ satisfies $f(x) \sim d x^{\alpha+\beta-1} \mathrm{e}^{-c x^{\beta}}$ as $x \rightarrow \infty$. Then the tail and the density of an i.i.d. sum satisfy

$$
\begin{aligned}
\overline{F^{* n}}(x)=\mathbb{P}\left(S_{n}>x\right) & \sim k(n) x^{\alpha(n)} \mathrm{e}^{-c(n) x^{\beta}}, \\
f^{* n}(x) & \sim \beta c(n) k(n) x^{\alpha(n)+\beta-1} \mathrm{e}^{-c(n) x^{\beta}},
\end{aligned}
$$

where $c(n)=c / n^{\beta-1}, \alpha(n)=n \alpha+(n-1) \beta / 2$, and

$$
k(n)=\frac{d^{n}}{\beta c}\left[\frac{2 \pi}{\beta(\beta-1) c}\right]^{(n-1) / 2} n^{\frac{1}{2}(\beta-n(2 \alpha+\beta)-1)} .
$$

Proof. We use induction. The statement is trivial for $n=1$, so assume it is proved for $n-1$. Taking $F_{1}=F, F_{2}=F^{*(n-1)}$ and applying Theorem $3.1 \mathrm{im}$ plies the result, and provides recurrences for $c(n), \alpha(n)$, and $k(n)$. To be specific, say that the $F_{i}$ distributions have densities $f_{i}$ like

$$
f_{i}(x) \sim d_{i}(n) x^{\alpha_{i}(n)+\beta-1} \mathrm{e}^{-c_{i}(n) x^{\beta}}, \quad i=1,2 .
$$

As $F_{1}=F$ is fixed, we simply have $c_{1}(n)=c, d_{1}(n)=d, \alpha_{1}(n)=\alpha$, and for $F_{2}=F^{*(n-1)}$ the induction hypothesis gives us

$$
c_{2}(n)=\frac{c}{(n-1)^{\beta-1}}, \quad d_{2}(n)=\beta c_{2}(n-1) k(n-1), \quad \alpha_{2}(n)=\alpha(n-1) .
$$

We extend the notation of Theorem 3.1 in an obvious way, for example we define $\eta(n)=c_{1}(n)^{1 /(\beta-1)}+c_{2}(n)^{1 /(\beta-1)}$. These simplify to

$$
\eta(n)=\frac{n c^{1 /(\beta-1)}}{n-1}, \quad \theta_{1}(n)=\frac{1}{n}, \quad \theta_{2}(n)=\frac{n-1}{n}, \quad \kappa(n)=\frac{n^{\beta-1}}{\beta c} .
$$

So $c(n)=c_{1}(n) \theta_{1}(n)^{\beta}+c_{2}(n) \theta_{2}(n)^{\beta}=c / n^{\beta}+c(n-1) / n^{\beta}=c / n^{\beta-1}$. Also, we have $\alpha(n)=\alpha_{1}(n)+\alpha_{2}(n)+\beta / 2=n \alpha+(n-1) \beta / 2$. 
The last recursion is less simple. We need the $\sigma$ constants:

$$
\sigma_{1}^{2}(n)=\frac{\beta c n^{-\beta}}{\beta-1}, \quad \sigma_{2}^{2}(n)=\frac{\beta c(n-1) n^{-\beta}}{\beta-1}, \quad \sigma^{2}(n)=\frac{\beta c(n-1) n^{-\beta-1}}{\beta-1} .
$$

Setting $k(1)=d /(\beta c)$, we get for $n \geqslant 2$

$$
\begin{aligned}
k(n) & =d_{1}(n) d_{2}(n) \theta_{1}(n)^{\alpha_{1}(n)} \theta_{2}(n)^{\alpha_{2}(n)} \kappa(n) \eta(n)^{1-\beta}\left(2 \pi \sigma(n)^{2}\right)^{1 / 2} / \beta \\
& =\left[\frac{2 \pi}{\beta(\beta-1) c}\right]^{1 / 2} d(n-1)^{\alpha(n-1)+\frac{1}{2}(\beta(n-2)+1)} n^{-\alpha n-\frac{1}{2} \beta(n-1)-\frac{1}{2}} k(n-1) \\
& =\frac{d^{n}}{\beta c}\left[\frac{2 \pi}{\beta(\beta-1) c}\right]^{(n-1) / 2} \prod_{\ell=2}^{n}(\ell-1)^{\alpha(\ell-1)+\frac{1}{2}(\beta(\ell-2)+1)} \ell^{-\alpha \ell-\frac{1}{2} \beta(\ell-1)-\frac{1}{2}} \\
& =\frac{d^{n}}{\beta c}\left[\frac{2 \pi}{\beta(\beta-1) c}\right]^{(n-1) / 2} n^{\frac{1}{2}(\beta-n(2 \alpha+\beta)-1)} .
\end{aligned}
$$

Note that $(B . T 0)$ is already given in Rootzén [16] (see his equations (6.1)(6.2)). We point out later that the assumptions on the density can be relaxed to $\bar{F}(x) \sim k x^{\alpha} \mathrm{e}^{-c x^{\beta}}$, where $k=d / c \beta$.

\section{LIGHT-TAILED SUMS}

We now proceed to the set-up of BKR and first introduce some terminology related to the densities of the form $f(x) \sim \gamma(x) \mathrm{e}^{-\psi(x)}$. The main assumption is that the function $\psi$ is non-negative, convex, $C^{2}$, and its first order derivative is denoted by $\lambda$. Further, it is supposed that

$$
\lim _{x \rightarrow \infty} \lambda(x)=\infty
$$

$\lambda^{\prime}$ is ultimately positive, and $1 / \sqrt{\lambda^{\prime}}$ is self-neglecting, i.e., as $x \rightarrow \infty$,

$$
\lambda^{\prime}\left(x+y / \sqrt{\lambda^{\prime}(x)}\right) \sim \lambda^{\prime}(x) .
$$

A function $\gamma$ is called flat for $\psi$ if locally uniformly on bounded $y$-intervals

$$
\lim _{x \rightarrow \infty} \frac{\gamma\left(x+y / \sqrt{\lambda^{\prime}(x)}\right)}{\gamma(x)}=1 .
$$

Similar conventions apply to functions denoted by $\psi_{1}, \psi_{2}$, etc. For the Weibull case,

$$
\psi(x)=a x^{\beta}, \quad \lambda(x)=a \beta x^{\beta-1}, \quad \gamma(x)=\lambda(x),
$$

and so (4.2) and (4.3) are satisfied. Examples beyond Weibull-like distributions are $\psi(x)=x \log x$ and $\psi(x)=\mathrm{e}^{a x}, a>0$. 
Define the class $\mathcal{H}(\gamma, \psi)$ as the class of all distributions $F$ having a density of the form $\gamma(x) \mathrm{e}^{-\psi(x)}$, where $\psi$ is as above, and $\gamma$ is a measurable function which is flat for $\psi$, and let $\overline{\mathcal{H}}(\gamma, \psi)$ be the class of distributions $F$ satisfying $\bar{F}(x) \sim$ $\gamma(x) \mathrm{e}^{-\psi(x)} / \lambda(x)$.

THEOREM 4.1. (i) $\mathcal{H}(\gamma, \psi) \subseteq \overline{\mathcal{H}}(\gamma, \psi)$.

(ii) Assume $F_{1} \in \mathcal{H}\left(\gamma_{1}, \psi_{1}\right), F_{2} \in \mathcal{H}\left(\gamma_{2}, \psi_{2}\right)$. Then $F_{1} * F_{2} \in \mathcal{H}(\gamma, \psi)$, where $\gamma, \psi$ are determined by first solving

$$
q_{1}+q_{2}=x, \quad \lambda_{1}\left(q_{1}\right)=\lambda_{2}\left(q_{2}\right)
$$

for $q_{1}=q_{1}(x), q_{2}=q_{2}(x)$, and next letting $\psi(x)=\psi_{1}\left(q_{1}\right)+\psi_{2}\left(q_{2}\right)$,

$$
\gamma(x)=\sqrt{\frac{2 \pi \lambda^{\prime}(x)}{\lambda_{1}^{\prime}\left(q_{1}\right) \lambda_{2}^{\prime}\left(q_{2}\right)}} \gamma_{1}\left(q_{1}\right) \gamma_{2}\left(q_{2}\right),
$$

where $\lambda(x)=\psi^{\prime}(x)=\lambda_{1}\left(q_{1}\right)=\lambda_{2}\left(q_{2}\right)$.

(iii) Let $F_{1} \in \overline{\mathcal{H}}\left(\gamma_{1}, \psi_{1}\right), F_{2} \in \overline{\mathcal{H}}\left(\gamma_{2}, \psi_{2}\right)$. Then there exists $H_{i} \in \mathcal{H}\left(\gamma_{i}, \psi_{i}\right)$, $H_{i} \in \operatorname{GMDA}\left(1 / \lambda_{i}\right)$, and

$$
\bar{H}_{i}(x) \sim \bar{F}_{i}(x), \quad \overline{H_{1} * H_{2}}(x) \sim \overline{F_{1} * F_{2}}(x) .
$$

Moreover, $F_{1} * F_{2} \in \overline{\mathcal{H}}(\gamma, \psi)$ with $\gamma, \psi$ as in (ii), and $F_{1} * F_{2} \in \operatorname{GMDA}(1 / \lambda)$.

The proof of Theorem 4.0 is given in the Appendix in Section W. Part (ii) is in BKR, here slightly reformulated, and a number of examples in BKR can be obtained as corollaries of this theorem.

REMARK 4.1. Letting $\tau(y)=\lambda_{1}^{\leftarrow}(y)+\lambda_{2}^{\leftarrow}(y)$, we can write the solution of (4.4) in the form

$$
q_{1}(x)=\lambda_{1}^{\leftarrow}\left(\tau^{\leftarrow}(x)\right), \quad q_{2}(x)=\lambda_{2}^{\leftarrow}\left(\tau^{\leftarrow}(x)\right)
$$

(here $\cdot \leftarrow$ means the functional inverse).

\section{BOUNDS}

There are easy upper- and lower-tail bounds for Weibull sums in terms of the incomplete gamma function $\Gamma(\alpha, x)=\int_{x}^{\infty} t^{\alpha-1} \mathrm{e}^{-t} \mathrm{~d} t$ when $\beta>1$, that in their simplest form just come from thinking about $p$-norms $\|\mathbf{y}\|_{p}=\left(\left|y_{1}\right|^{p}+\ldots+\left|y_{n}\right|^{p}\right)^{1 / p}$ and the fact that if $Y$ is standard exponential, then $Y^{1 / \beta}$ is Weibull with tail $\mathrm{e}^{-x^{\beta}}$.

Proposition 5.1. Let $X$ have density $\beta k^{\gamma / \beta} x^{\gamma-1} \mathrm{e}^{-k x^{\beta}} / \Gamma(\gamma / \beta), x>0$, where $k>0, \beta \geqslant 1$, and $\gamma>0$. Then

$$
\frac{\Gamma\left(n \gamma / \beta, k x^{\beta}\right)}{\Gamma(n \gamma / \beta)} \leqslant \mathbb{P}\left(X_{1}+\ldots+X_{n}>x\right) \leqslant \frac{\Gamma\left(n \gamma / \beta, k x^{\beta} / n^{\beta-1}\right)}{\Gamma(n \gamma / \beta)} .
$$


Pro of. An $X$ with the given density has the same distribution as $(Y / k)^{1 / \beta}$, where $Y$ is $\operatorname{Gamma}(\alpha, 1)$ with density $y^{\alpha-1} \mathrm{e}^{-y} / \Gamma(\alpha)$, and $\alpha=\gamma / \beta$. Therefore,

$$
X_{1}^{\beta}+\ldots+X_{n}^{\beta}=\|\mathbf{X}\|_{\beta}^{\beta} \stackrel{d}{=}\|\mathbf{Y} / k\|_{1}=Y_{1} / k+\ldots+Y_{n} / k,
$$

where $Y_{1}, \ldots, Y_{n}$ are i.i.d. Gamma $(\alpha, 1)$. From the Jensen and Hölder inequalities it follows for $p \geqslant 1$ and $\mathbf{x} \in \mathbb{R}^{n}$ that

$$
\|\mathbf{x}\|_{p} \leqslant\|\mathbf{x}\|_{1} \leqslant\|\mathbf{x}\|_{p} n^{1-1 / p} .
$$

Hence, since $\|\mathbf{Y}\|_{1}=Y_{1}+\ldots+Y_{n}$ is $\operatorname{Gamma}(n \alpha, 1)$ with tail $\Gamma(n \alpha, y) / \Gamma(n \alpha)$, we have for any $x>0$

$$
\begin{aligned}
\mathbb{P}\left(X_{1}+\ldots+X_{n}>x\right) & =\mathbb{P}\left(\|\mathbf{X}\|_{1}>x\right) \\
& \leqslant \mathbb{P}\left(\|\mathbf{X}\|_{\beta}^{\beta}>x^{\beta} / n^{\beta-1}\right)=\mathbb{P}\left(\|\mathbf{Y}\|_{1}>k x^{\beta} / n^{\beta-1}\right),
\end{aligned}
$$

and similarly for the lower bound.

The (upper) incomplete gamma function $\Gamma(\alpha, x)$ appearing here is available in most standard software, but note that an even simpler lower bound comes from $\Gamma(\alpha, x) \geqslant x^{\alpha-1} \mathrm{e}^{-x}$ for $x>0$ when $\alpha=\gamma / \beta \geqslant 1$, respectively, $\Gamma(\alpha, x) \geqslant$ $x^{\alpha-1} \mathrm{e}^{-x}(x /(x+1-\alpha))$ when $\alpha \in(0,1)$. Moreover, observe that $X$ with the density given in Proposition 5.1 has tail probability

$$
\bar{F}_{X}(x)=\mathbb{P}(X>x)=\frac{\Gamma\left(\gamma / \beta, k x^{\beta}\right)}{\Gamma(\gamma / \beta)} .
$$

Hence, appealing to the fact that $\Gamma(\alpha, x) \sim x^{\alpha-1} \mathrm{e}^{-x}$ as $x \rightarrow \infty$, we see that the upper bound in Proposition 5.$]$ is asymptotically

$$
\frac{\Gamma(\gamma / \beta)^{n}}{\Gamma(n \gamma / \beta)} n^{n \gamma / \beta-1} k^{n-1}\left(\frac{x}{n}\right)^{\beta(n-1)} \bar{F}_{X}(x / n)^{n} .
$$

When $\gamma=\beta$ (the ordinary Weibull case), the ratio of this upper bound to the true asymptotic form for $\mathbb{P}\left(X_{1}+\ldots+X_{n}>x\right)$ is

$$
\frac{n^{(n-1 / 2)}}{(n-1) !}\left[\frac{(\beta-1)}{2 \pi \beta}\right]^{(n-1) / 2} k^{(n-1) / 2}\left(\frac{x}{n}\right)^{\beta(n-1) / 2},
$$

so the upper bound is out only by a polynomial factor in $x$, which indicates it is close to the true probability on a logarithmic scale. More precisely, writing $U(x)$ for the upper bound and $P(x)$ for the true probability, it follows trivially that $x^{-1} \log (U(x)) \sim x^{-1} \log (P(x))$ as $x \rightarrow \infty$.

It is straightforward to extend Proposition $[5$.$] to the following slightly more$ general form. 
Proposition 5.2. Let $\left\{X_{i}\right\}_{i=1}^{n}$ be independent random variables with density $\beta k^{\gamma_{i} / \beta} x^{\gamma_{i}-1} \mathrm{e}^{-k x^{\beta}} / \Gamma\left(\gamma_{i} / \beta\right), x>0$, where $k>0, \beta \geqslant 1$, and $\gamma_{i}>0$ for $i=1, \ldots, n$. Then with $\gamma_{0}=\sum_{i=1}^{n} \gamma_{i}$ we have

$$
\frac{\Gamma\left(\gamma_{0} / \beta, k x^{\beta}\right)}{\Gamma\left(\gamma_{0} / \beta\right)} \leqslant \mathbb{P}\left(X_{1}+\ldots+X_{n}>x\right) \leqslant \frac{\Gamma\left(\gamma_{0} / \beta, k x^{\beta} / n^{\beta-1}\right)}{\Gamma\left(\gamma_{0} / \beta\right)} .
$$

\section{MOMENT-GENERATING FUNCTIONS AND THE EXPONENTIAL FAMILY}

In this section, we assume that $X \sim F$ has the tail asymptotics $\gamma(x) \mathrm{e}^{-x^{\beta}} / \lambda(x)$ for some $\beta>1$, where $\lambda(x)=\beta x^{\beta-1}$. Define

$$
\widehat{F}[\theta]=\mathbb{E}\left[\mathrm{e}^{\theta X}\right]=\int_{-\infty}^{\infty} \mathrm{e}^{\theta z} F(\mathrm{~d} z), \quad F_{\theta}(\mathrm{d} z)=\frac{\mathrm{e}^{\theta z}}{\widehat{F}[\theta]} F(\mathrm{~d} z),
$$

where expectations with respect to $F_{\theta}$ will be denoted by $\mathbb{E}_{\theta}[\cdot]$. Determining the asymptotics of $\widehat{F}[\theta]$ and characteristics of the exponential family like their moments is easier when taking $\theta=\lambda(x)$. For a general $\theta$, one then just has to substitute $x=\lambda^{\leftarrow}(\theta)$ in the following result.

Proposition 6.1. As $x \rightarrow \infty$, we have

$$
\begin{aligned}
\widehat{F}[\lambda(x)] & \sim \sqrt{\frac{2 \pi}{\lambda^{\prime}(x)}} \gamma(x) \mathrm{e}^{(\beta-1) x^{\beta}}, \\
\mathbb{E}_{\lambda(x)} X & \sim x
\end{aligned}
$$

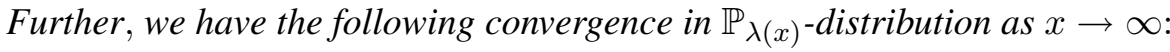

$$
\sqrt{\lambda^{\prime}(x)}(X-x)=\sqrt{\beta(\beta-1) x^{\beta-2}}(X-x) \stackrel{\mathcal{D}}{\longrightarrow} N(0,1) .
$$

P r o o f. Suppose for simplicity that $X$ is non-negative. Using Proposition 3.2 in BKR, we can assume without loss of generality that $\gamma \in C^{\infty}$. By Theorem 4.d] we obtain $\bar{F}(x) \sim \bar{H}(x)$, where $H$ has the density $\gamma(z) \mathrm{e}^{-z^{\beta}}$ for $z \geqslant 0$. It follows easily from our proof below that $\mathbb{E}\left[X^{k} \mathrm{e}^{\lambda(x) X}\right] \sim \mathbb{E}\left[X_{*}^{k} \mathrm{e}^{\lambda(x) X_{*}}\right]$ for $k \geqslant 0$ with $X_{*} \sim H$, so we assume without loss of generality that $F$ has the density $f(z)=$ $\gamma(z) \mathrm{e}^{-z^{\beta}}$ for $z \geqslant 0$. Lastly, we have $\gamma(x)=o\left(\mathrm{e}^{c x}\right)$ for any $c>0$, as

$$
\lim _{x \rightarrow \infty} \frac{\gamma^{\prime}(x)}{\sqrt{\lambda^{\prime}(x)} \gamma(x)}=0 .
$$


For $g(z)=z^{k} \mathrm{e}^{\lambda(x) z}$, with $k \geqslant 0$, it follows by integration by parts that

$$
\begin{aligned}
\mathbb{E}\left[X^{k} \mathrm{e}^{\lambda(x) X}\right] & =g(0)+\int_{0}^{\infty} g^{\prime}(z) \bar{F}(z) \mathrm{d} z \\
& =\mathbb{I}\{k=0\}+\int_{0}^{c_{1} x} g^{\prime}(z) \bar{F}(z) \mathrm{d} z+\int_{c_{1} x}^{\infty} g^{\prime}(z) \bar{F}(z) \mathrm{d} z \\
& =O\left(\mathrm{e}^{\tilde{c}_{1} x^{\beta}}\right)+\int_{c_{1} x}^{\infty}\left[k z^{k-1}+\lambda(x) z^{k}\right] \mathrm{e}^{\lambda(x) z} \frac{\gamma(z)}{\lambda(z)} \mathrm{e}^{-z^{\beta}} \mathrm{d} z
\end{aligned}
$$

for any $0<c_{1}<\tilde{c}_{1}<1$ sufficiently small.

Consider integrals of the form $\int_{c_{1} x}^{\infty} z^{k} \mathrm{e}^{\lambda(x) z} \gamma(z) \mathrm{e}^{-z^{\beta}} \mathrm{d} z$ and note that the global maximum of the exponent $\lambda(x) z-z^{\beta}$ is at $z=x$. We use the substitution $z=x+y / \lambda(x)$ (similar to those in Sections $\square$ and B) and note that

$$
\lambda(x) z-z^{\beta} \sim(\beta-1) x^{\beta}-\frac{y^{2} \lambda^{\prime}(x)}{2 \lambda(x)^{2}} .
$$

Therefore, for any $D>0$ we have, as $x \rightarrow \infty$,

$$
\begin{aligned}
& \int_{c_{1} x}^{\infty} z^{k} \frac{\gamma(z)}{\lambda(z)} \mathrm{e}^{\lambda(x) z-z^{\beta}} \mathrm{d} z \sim \int_{x-D / \lambda(x)}^{x+D / \lambda(x)} z^{k} \frac{\gamma(z)}{\lambda(z)} \mathrm{e}^{\lambda(x) z-z^{\beta}} \mathrm{d} z \\
\sim & \int_{-D}^{D}\left(x+\frac{y}{\lambda(x)}\right)^{k} \frac{\gamma(x+y / \lambda(x))}{\lambda(x+y / \lambda(x))} \exp \left\{(\beta-1) x^{\beta}-\frac{y^{2} \lambda^{\prime}(x)}{2 \lambda(x)^{2}}\right\} \frac{1}{\lambda(x)} \mathrm{d} y \\
\sim & x^{k} \frac{\gamma(x)}{\lambda(x)^{2}} \mathrm{e}^{(\beta-1) x^{\beta}} \int_{-D}^{D} \exp \left\{-\frac{y^{2} \lambda^{\prime}(x)}{2 \lambda(x)^{2}}\right\} \mathrm{d} y \sim \sqrt{\frac{2 \pi}{\lambda^{\prime}(x)}} x^{k} \frac{\gamma(x)}{\lambda(x)} \mathrm{e}^{(\beta-1) x^{\beta}}
\end{aligned}
$$

where the replacement of the limits $\pm D$ by $\pm \infty$ follows from $\lambda^{\prime}(x) / \lambda(x)^{2} \rightarrow 0$. Combining this integral asymptotic with (6.5)), we get

$$
\begin{aligned}
\mathbb{E}\left[X^{k} \mathrm{e}^{\lambda(x) X}\right]= & O\left(\mathrm{e}^{\tilde{c}_{1} x^{\beta}}\right)+k \int_{c_{1} x}^{\infty} z^{k-1} \frac{\gamma(z)}{\lambda(z)} \mathrm{e}^{\lambda(x) z-z^{\beta}} \mathrm{d} z \\
& +\lambda(x) \int_{c_{1} x}^{\infty} z^{k} \frac{\gamma(z)}{\lambda(z)} \mathrm{e}^{\lambda(x) z-z^{\beta}} \mathrm{d} z
\end{aligned}
$$

that is

$$
\mathbb{E}\left[X^{k} \mathrm{e}^{\lambda(x) X}\right]=O\left(\mathrm{e}^{\tilde{c}_{1} x^{\beta}}\right)+\sqrt{\frac{2 \pi}{\lambda^{\prime}(x)}} \gamma(x) \mathrm{e}^{(\beta-1) x^{\beta}}\left(x^{k}+\frac{k}{\lambda(x)} x^{k-1}\right)
$$


or to take only the largest term,

$$
\mathbb{E}\left[X^{k} \mathrm{e}^{\lambda(x) X}\right] \sim \sqrt{\frac{2 \pi}{\lambda^{\prime}(x)}} \gamma(x) x^{k} \mathrm{e}^{(\beta-1) x^{\beta}} \quad \text { as } x \rightarrow \infty .
$$

Consequently, we obtain easily (6.1) and (6.2).

Next, we show the asymptotic normality. By the above arguments, we assume for simplicity that $F$ has density $f(z)=\gamma(z) \mathrm{e}^{-z^{\beta}}$ for all $z>0$. Similarly, writing instead $z=x+y / \sqrt{\lambda^{\prime}(x)}$, we have

$$
\lambda(x) z-z^{\beta} \sim(\beta-1) x^{\beta}-\frac{y^{2}}{2} .
$$

For some $D<\min (0, v)$ we obtain

$$
\begin{aligned}
\int_{x+D / \sqrt{\lambda^{\prime}(x)}}^{x+v / \sqrt{\lambda^{\prime}(x)}} & \gamma(z) \exp \left\{\lambda(x) z-z^{\beta}\right\} \mathrm{d} z \\
& \sim \frac{1}{\sqrt{\lambda^{\prime}(x)}} \int_{D}^{v} \gamma\left(x+y / \sqrt{\lambda^{\prime}(x)}\right) \exp \left\{(\beta-1) x^{\beta}-\frac{y^{2}}{2}\right\} \mathrm{d} y \\
& \sim \frac{1}{\sqrt{\lambda^{\prime}(x)}} \gamma(x) \mathrm{e}^{(\beta-1) x^{\beta}} \int_{D}^{v} \exp \left\{-\frac{y^{2}}{2}\right\} \mathrm{d} y .
\end{aligned}
$$

Hence, letting $D \rightarrow-\infty$ yields

$$
\mathbb{E}\left[\mathrm{e}^{\lambda(x) X} ; \sqrt{\lambda^{\prime}(x)}(X-x) \leqslant v\right] \sim \sqrt{\frac{2 \pi}{\lambda^{\prime}(x)}} \gamma(x) \mathrm{e}^{(\beta-1) x^{\beta}} \Phi(v) .
$$

Dividing by (6.ل gives $\mathbb{P}_{\lambda(x)}\left(\sqrt{\lambda^{\prime}(x)}(X-x) \leqslant v\right) \rightarrow \Phi(v)$, which is (6.3)).

REMARK 6.1. Asymptotic normality for the general case $\bar{F}(x)=\mathrm{e}^{-\psi(x)}$ similar to the result of Proposition 6.] is derived in [8].

REMARK 6.2. The BKR method of proof is modelled after the standard proof of the saddlepoint approximation: exponential change of measure using estimates of the above type. One has

$$
\mathbb{P}\left(S_{n}>x\right)=\widehat{F}[\theta]^{n} \mathbb{E}_{\theta}\left[\mathrm{e}^{-\theta S_{n}} ; S_{n}>x\right],
$$

and it should take $\theta$ such that $\mathbb{E}_{\theta} S_{n}=x$, i.e. $\theta=\lambda(x / n)$. The approximate normality of $\left(X_{1}, \ldots, X_{n}\right)$ implies that $S_{n}$ is approximately normal $\left(x, n / \lambda^{\prime}(x / n)\right)$. So, one can compute

$$
\mathbb{E}_{\lambda(x / n)} \exp \left\{-a \sqrt{\lambda^{\prime}(x / n) / n} S_{n}\right\}
$$


for any fixed $a$ but $\theta=\lambda(x / n)$ is of a different order than $\sqrt{\lambda^{\prime}(x / n) / n}$. Therefore (as for the saddlepoint approximation), a sharper CLT is needed, and this is maybe the most demanding part of the BKR approach.

\section{COMPOUND POISSON SUMS}

We consider here $S_{N}=X_{1}+\ldots+X_{N}$, where $N$ is $\operatorname{Poisson}(\mu)$ and independent of $X_{1}, X_{2}, \ldots$, with $X_{i} \sim \operatorname{Weibull}(\beta)$. The asymptotics of $\mathbb{P}\left(S_{N}>x\right)$ are important in many applications, for example, actuarial sciences [3], and can be investigated by using classical saddlepoint techniques. The relevant asymptotic is the classical Esscher approximation:

$$
\mathbb{P}\left(S_{N}>x\right) \sim \frac{\left(\widehat{F_{S_{N}}}[\theta]-\mathrm{e}^{-\mu}\right) \exp \{-\theta x\}}{\theta \sigma_{c}(\theta)} B_{0}(\ell),
$$

where $\theta$ is the solution to $\mu \widehat{F}^{\prime}[\theta]=x$, and $\widehat{F_{S_{N}}}[\theta]=\exp \{\mu(\widehat{F}[\theta]-1)\}, B_{0}(l)=$ $l \mathrm{e}^{l^{2} / 2}(1-\Phi(l)) \rightarrow(2 \pi)^{-1 / 2}, \sigma_{c}^{2}(\theta)=\mu F^{\prime \prime}[\theta]$, and $\ell=\theta \sigma_{c}(\theta)$. See (7.1.10) in [114], where also further refinements and variants are given. The issue with implementing $(\mathbb{D} \mathbb{C})$ is that we do not usually have access to $\widehat{F}[\theta]$; note, Mathematica can derive $\widehat{F}[\theta]$ when $\beta=1.5,2$, or 3 .

For standard Weibull $(\beta)$ variables, (6.1) simplifies to

$$
\widehat{F}[t] \sim \sqrt{\frac{2 \pi \beta^{1 /(1-\beta)}}{\beta-1}} t^{\beta /(2(\beta-1))} \exp \left\{(\beta-1)(t / \beta)^{\beta /(\beta-1)}\right\}=: \widetilde{F}[t] .
$$

Unfortunately, $\widehat{F_{S_{N}}}[t] \nsim \exp \{\mu(\widetilde{F}[t]-1)\}$, though $\widehat{F_{S_{N}}}[t] \approx_{\log } \exp \{\mu(\widetilde{F}[t]-1)\}$, where the notation $h_{1}(x) \approx_{\log } h_{2}(x)$ means that $\log h_{1}(x) / \log h_{2}(x) \rightarrow 1$.

One can select the $\theta$ which solves $\mu \widetilde{F}^{\prime}[\theta]=x$; however, it seems this must be done numerically. An alternative can be the asymptotic forms for $\widehat{F}^{(k)}$ from (6.7). Take

$$
\widehat{F}^{(k)}[\theta]=\mathbb{E}\left[X^{k} \mathrm{e}^{\theta X}\right] \sim y^{k} \widehat{F}[\theta] \quad \text { for } k \in \mathbb{N}
$$

where we have written $\theta=\lambda(y)$ as in Section 6. Thus, if we set $\theta$ as the solution to $\mu y \widetilde{F}[\lambda(y)]=x$, then we get

$$
y=2^{-1 / \beta}\left[\frac{\beta+2}{(\beta-1) \beta} \mathcal{W}\left(\frac{(\beta-1) \beta}{\beta+2}\left(\frac{2^{1 / \beta+1 / 2} x}{c_{1}}\right)^{2 \beta /(\beta+2)}\right)\right]^{1 / \beta}
$$

where $\mathcal{W}$ is the Lambert $W$-function and $c_{1}=\mu \sqrt{2 \pi} \beta / \sqrt{(\beta-1) \beta}$. 
With this choice of $\theta$, we can say that $\widehat{F}^{(k)}[\theta] \sim x y^{k-1}$, so $\sigma_{c}^{2}(\theta) \sim \mu x y$ and $\ell \sim \lambda(y) \sqrt{\mu x y}$, and substituting this into (पDT) gives us

$$
\mathbb{P}\left(S_{N}>x\right) \approx_{\log } \frac{\mathrm{e}^{-\mu}(\exp \{\mu x / y\}-1) \exp \{-\theta x\}}{\lambda(y) \sqrt{\mu x y}} B_{0}(\ell) .
$$

Preliminary numerical work indicates that (L.4) is not particularly accurate in the whole range of relevant parameters. The problem derives from the fact we only have log-asymptotics for $\widehat{F_{S_{N}}}[\theta]$; finding more accurate asymptotics is left for future work.

A further interesting extension could be the asymptotic form of $\mathbb{P}(Z(t)>x)$, $Z$ being a Lévy process where the Lévy measure has tail $\gamma(x) \mathrm{e}^{-\psi(x)}$.

\section{THE EXPONENTIAL CLASS OF DISTRIBUTIONS}

For $F \in \operatorname{GMDA}(e)$ in the previous sections we have discussed the case where $e(x)=1 / \lambda(x)$ with

$$
\lim _{x \rightarrow \infty} e(x)=0 .
$$

If $\lim _{x \rightarrow \infty} e(x)=\infty$, then $F$ is long-tailed in the sense that $\bar{F}(x-y) \sim \bar{F}(x)$ for any fixed $y$. Convolutions of distributions that are long-tailed are well understood. The intermediate case is

$$
\lim _{x \rightarrow \infty} e(x)=1 / \gamma, \quad \gamma>0
$$

For such $F$ we have

$$
\bar{F}(x+s) \sim \mathrm{e}^{-\gamma s} \bar{F}(x), \quad x \rightarrow \infty,
$$

for any $s \in \mathbb{R}$, which is also denoted as $F \in \mathcal{L}(\gamma)$. Note in passing that any distribution $F \in \operatorname{GMDA}(e)$ with upper endpoint infinity satisfies (see, e.g., [15], Proposition 1.4)

$$
\bar{F}(x) \sim \bar{H}(x)=C \exp \left\{-\int_{0}^{x} \frac{1}{u(t)} \mathrm{d} t\right\}, \quad x \rightarrow \infty,
$$

for some $C>0$, where $u$ is absolutely continuous with respect to Lebesgue measure, with density $u^{\prime}$ satisfying $\lim _{x \rightarrow \infty} u^{\prime}(x)=0$. Such $H$ is commonly referred to as a von Mises distribution.

It is well known (see [10], [17]) that the class of distributions $\mathcal{L}(\gamma)$ is closed under convolution. In the particular case that the $X_{i}$ have tails

$$
\bar{F}_{i}(x)=\ell_{i}(x) x^{\gamma_{i}-1} \mathrm{e}^{-k x^{\beta}}, \quad 1 \leqslant i \leqslant n,
$$


$\ell_{i}$ 's being positive slowly varying functions and $\beta=1, \gamma_{i}>0, i \leqslant n, k>0$, we have in view of Theorem 2.1 in [13] (see also Theorem 6.4 (ii) in [I])

$$
\mathbb{P}\left(S_{n}>x\right) \sim \frac{k^{n-1}}{\Gamma\left(\gamma_{0}\right)} x^{\gamma_{0}-1} \prod_{i=1}^{n} \ell_{i}(x) \mathrm{e}^{-k x^{\beta}},
$$

where $\gamma_{0}=\sum_{i=1}^{n} \gamma_{i}$. If (8.3) holds with $\beta>1$, then for non-negative $X_{i}$ 's, using the $\beta$-norm argument, we have, as in Section [1,

$$
\mathbb{P}\left(S_{n}>x\right) \leqslant \mathbb{P}\left(X_{1}^{\beta}+\ldots+X_{n}^{\beta}>x^{\beta} / n^{\beta-1}\right)
$$

for any $x>0$. Since $\mathbb{P}\left(X_{1}^{\beta}>x\right) \sim \ell_{i}\left(x^{1 / \beta}\right) x^{\left(\gamma_{i}-1\right) / \beta} \mathrm{e}^{-k x}$, by (8.3) and Theorem 4.1 we have

$$
\ln \mathbb{P}\left(S_{n}>x\right) \sim \ln \mathbb{P}\left(X_{1}^{\beta}+\ldots+X_{n}^{\beta}>x^{\beta} / n^{\beta-1}\right) \sim k n(x / n)^{\beta},
$$

and thus the upper bound in (8.4) is logarithmic asymptotically exact.

\section{APPLICATIONS TO MONTE CARLO SIMULATION}

In this section, we write $h_{1}(x) \approx_{\log } h_{2}(x)$ if $\log h_{1}(x) / \log h_{2}(x) \rightarrow 1$, and $\leqslant \log$ if the lim sup of the ratio of logarithms is at most one, and we take the summands to have a density like $\gamma(x) \mathrm{e}^{-x^{\beta}}$ as $x \rightarrow \infty$.

Algorithms for tails $\mathbb{P}\left(S_{n}>x\right)$ with large $x$ are one of the traditional objects of study of the rare-event simulation literature. An estimator is an r.v. $Z(x)$ with $\mathbb{E} Z(x)=\mathbb{P}\left(S_{n}>x\right)$ and its efficiency is judged by ratios of the form $r_{p}(x)=$ $\mathbb{E} Z(x)^{2} / \mathbb{P}\left(S_{n}>x\right)^{p}$. The estimator will improve upon crude Monte Carlo simulation if $r_{1}(x) \rightarrow 0$ as $x \rightarrow \infty$. It is said to have bounded relative error if $r_{2}(x)$ stays bounded as $x \rightarrow \infty$, and to exhibit logarithmic efficiency if $r_{2-\varepsilon}(x) \rightarrow 0$ for all $\varepsilon>0$, which in turn will hold if $\mathbb{E} Z(x)^{2} \approx_{\log } \mathbb{P}\left(S_{n}>x\right)^{2}$. These two concepts are usually considered in some sense optimal. For a survey, see Chapters V and VI in [4].

The conventional light-tailed rare-event folklore says that a particular kind of importance sampling, exponential tilting, is often close to optimal. Here instead of $\mathbb{I}\left\{S_{n}>x\right\}$ one returns

$$
Z_{\theta}(x)=\mathbb{I}\left\{S_{n}>x\right\} \times L_{\theta}, \quad \text { where } L_{\theta}=\widehat{F}[\theta]^{n} \exp \left\{-\theta S_{n}\right\},
$$

and $X_{1}, \ldots, X_{n}$ are i.i.d. with density $f_{\theta}(y)=\mathrm{e}^{\theta y} f(y) / \widehat{F}(\theta)$ rather than the given density $f(x)$, and $\theta$ is chosen such that $\mathbb{E}_{\theta} X=x / n$, that is, $\theta=\lambda(x / n)$. The standard efficiency results do, however, require both $n \rightarrow \infty$ and $x \rightarrow \infty$ such that $n x \sim z$ for some $z>\mathbb{E} X$, and therefore do not deal with a fixed $n$, the object of this paper. It is believed that the scheme is still often close to optimal in this setting, 
but very few rigorous results in this direction have been formulated. We give one such in Proposition $\mathrm{QT} .1 \mathrm{~b}$ below.

One problem that arises is how to simulate from $f_{\theta}$. Proposition 6.1 tells us that $f_{\theta}$ is asymptotically normal with mean $x / n$ and variance $1 / \lambda^{\prime}(x / n)$ when $\theta=\lambda(x / n)$. So we simulate using acceptance-rejection with a moment-matched gamma distribution as a proposal, and our acceptance ratio will increase to one as $x \rightarrow \infty$. To be specific, we take a $\operatorname{Gamma}(a, b)$ proposal, which has a density $f_{a, b}(y) \propto y^{a-1} \mathrm{e}^{-b y}$, where $a=x^{2} \lambda^{\prime}(x / n) / n^{2}$, and $b=x \lambda^{\prime}(x / n) / n$. The reason we do not directly use the limiting normal distribution as a proposal is that the tail of the normal distribution is too light when $\beta \in(1,2)$.

REMARK 9.1. The acceptance ratio can be improved for small $x$ by searching locally for the optimal proposal, that is, the distribution with parameters

$$
\left(\mu^{*}, \sigma^{*}\right)=\underset{\mu, \sigma>0}{\arg \min } \max _{y \geqslant 0} \frac{f_{\lambda(x / n)}(y)}{f_{\operatorname{Prop}}\left(y ; \mu, \sigma^{2}\right)} .
$$

The asymptotic $(\mu, \sigma)=\left(x / n, 1 / \sqrt{\lambda^{\prime}(x / n)}\right)$ can be used as the initial search point. In experiments, it seems that the asymptotic variance is close to optimal, whereas some efficiency can be gained by adjusting the mean parameter.

PROPOSITION 9.1. The estimator $Z_{\theta}(x)$ exhibits logarithmic efficiency.

Pro of. We first note that

$$
\mathbb{E}_{\theta}\left[Z_{\theta}(x)^{2}\right]=\mathbb{E}_{\theta}\left[L_{\theta}^{2} ; S_{n}>x\right]=\mathbb{E}\left[L_{\theta} ; S_{n}>x\right] \leqslant \mathrm{e}^{-\theta x} \widehat{F}[\theta]^{n} \mathbb{P}\left(S_{n}>x\right) .
$$

By Corollary 3.$]$ and (6.T),

$$
\bar{F}^{* n}(x) \approx_{\log } \exp \left\{n(x / n)^{\beta}\right\}, \quad \widehat{F}[\lambda(x / n)]^{n} \approx_{\log } \exp \left\{n(\beta-1)(x / n)^{\beta}\right\} .
$$

From $\theta=\lambda(x / n)=\beta(x / n)^{\beta-1}$ we then get

$$
\begin{aligned}
\frac{\operatorname{Var}_{\theta}\left(Z_{\theta}(x)\right)}{\mathbb{P}\left(S_{n}>x\right)} & \leqslant \frac{\mathbb{E}_{\theta}\left[Z_{\theta}(x)^{2}\right]}{\mathbb{P}\left(S_{n}>x\right)} \\
& \leqslant \log \exp \left\{-\theta x+n(\beta-1)(x / n)^{\beta}+n(x / n)^{\beta}\right\} \\
& =\exp \left\{-\beta(x / n)^{\beta-1} x+n \beta(x / n)^{\beta}\right\}=1,
\end{aligned}
$$

completing the proof.

Some estimators based on conditional Monte Carlo ideas are discussed in [2], and efficiency properties derived in some special cases. The algorithms do improve upon crude Monte Carlo, though logarithmic efficiency is not obtained. The advantage is, however, that they are much easier implemented than the above exponential tilting scheme. The next two propositions extend results of [2] to more general tails. 
Proposition 9.2. Consider the conditional Monte Carlo estimator $Z_{\mathrm{Cd}}(x)$ $=\bar{F}\left(x-S_{n-1}\right)$ of $\mathbb{P}\left(S_{n}>x\right)$. Then $\lim \sup r_{p}(x)<\infty$ whenever $p<p_{n}$, where $p_{n}=n^{\beta-1} c_{n}$ with $c_{n}$ given by (Q.]) below. Here $p_{n}>1$.

Pr o o f. We have $\mathbb{E} Z_{\mathrm{Cd}}(x)^{2}=\int \bar{F}(x-y)^{2} f^{*(n-1)}(y) \mathrm{d} y$, where the asymptotics of the integral is covered by Theorem B.l. In the setting there, $c_{1}=2$, $c_{2}=1 /(n-1)^{\beta-1}$, which gives $\theta_{1}=1 /(1+\mu), \theta_{2}=\mu /(1+\mu)$, where $\mu=$ $2^{1 /(\beta-1)}(n-1)$. The result implies $\mathbb{E} Z_{\mathrm{Cd}}(x)^{2} \approx_{\log } \mathrm{e}^{-c_{n} x^{\beta}}$, where

$$
c_{n}=c_{1} \theta_{1}^{\beta}+c_{2} \theta_{2}^{\beta}=\frac{2+2^{\beta /(\beta-1)}(n-1)}{\left(1+2^{1 /(\beta-1)}(n-1)\right)^{\beta}} .
$$

Since $\mathbb{P}\left(S_{n}>x\right) \approx_{\log } \mathrm{e}^{-x^{\beta} / n^{\beta-1}}$, this implies the first assertion of the proposition. To see that $p_{n}>1$, note that for $a>1$

$$
n^{\beta-1} \frac{a^{\beta-1}+a^{\beta}(n-1)}{(1+a(n-1))^{\beta}}=\left[\frac{n a}{1+a(n-1)}\right]^{\beta-1}>\left[\frac{n a}{n a}\right]^{\beta-1}=1
$$

and take $a=2^{1 /(\beta-1)}$

We finally consider the so-called Asmussen-Kroese estimator

$$
Z_{\mathrm{AK}}(x)=n \bar{F}\left(M_{n-1} \vee\left(x-S_{n-1}\right)\right),
$$

where $M_{n-1}=\max \left(X_{1}, \ldots, X_{n-1}\right)$. It was initially developed in [5] with heavy tails in mind, but it was found empirically in [2] that it also provides some variance reduction for light tails, in fact more than $Z_{\mathrm{Cd}}(x)$. We have

Proposition 9.3. Consider the estimator $Z_{\mathrm{AK}}(x)$ of $\mathbb{P}\left(S_{n}>x\right)$ with $n=2$. Then $\lim \sup r_{p}(x)<\infty$ whenever $p<3 / 2$.

Proof. When $n=2$, we have $M_{n-1}=S_{n-1}=X_{1}$, and so the analysis splits into an $X_{1}>x / 2$ and an $X_{1} \leqslant 2$ part. The first is

$$
\begin{aligned}
\mathbb{E}\left[Z_{\mathrm{AK}}(x)^{2} ; X_{1}>x / 2\right] & =4 \int_{x / 2}^{\infty} \bar{F}(y)^{2} f(y) \mathrm{d} y \\
& \approx_{\log } \int_{x / 2}^{\infty} \mathrm{e}^{-2 y^{\beta}} \mathrm{e}^{-y^{\beta}} \mathrm{d} y \approx_{\log } \mathrm{e}^{-3 x^{\beta} / 2^{\beta}} .
\end{aligned}
$$

The second part is

$$
\begin{aligned}
\mathbb{E}\left[Z_{\mathrm{AK}}(x)^{2} ; X_{1} \leqslant x / 2\right] & =4 \int_{-\infty}^{x / 2} \bar{F}(x-y)^{2} f(y) \mathrm{d} y \\
& =4 \int_{x / 2}^{\infty} \bar{F}(y)^{2} f(x-y) \mathrm{d} y=4 I_{1}+4 I_{2},
\end{aligned}
$$


where $I_{1}$ is the integral over $[x / 2, a x)$, and $I_{2}$ is the one over $[a x, \infty)$. Here we take $a=(3 / 2)^{1 / \beta} / 2$; since $\beta>1$, we have $a<3 / 4<1$. Let further $b=a-1 / 2$. Then

$$
\begin{aligned}
& I_{2}=\int_{a x}^{\infty} \bar{F}(y)^{2} O(1) \mathrm{d} y \approx_{\log } \int_{a x}^{\infty} \mathrm{e}^{-2 x^{\beta}} O(1) \mathrm{d} y \approx_{\log } \mathrm{e}^{-2 a^{\beta} x^{\beta}}=\mathrm{e}^{-3 x^{\beta} / 2^{\beta}}, \\
& I_{1} \approx_{\log } \int_{x / 2}^{a x} \exp \left\{-2 y^{\beta}-(x-y)^{\beta}\right\}=\int_{0}^{b x} \exp \left\{-2(x / 2+z)^{\beta}-(x / 2-z)^{\beta}\right\} \mathrm{d} z .
\end{aligned}
$$

By convexity of $v \mapsto v^{\beta}$, we have

$$
(u+v)^{\beta}=u^{\beta}(1+v / u)^{\beta} \geqslant u^{\beta}(1+\beta v / u)=u^{\beta}+\beta v u^{\beta-1}
$$

for $u>0$ and $-u<v<\infty$. Taking $u=x / 2$ gives

$$
I_{2} \leqslant \log \int_{0}^{b x} \exp \left\{-3 x^{\beta} / 2^{\beta}-\beta z(x / 2)^{\beta-1}\right\} \mathrm{d} z=\mathrm{e}^{-3 x^{\beta} / 2^{\beta}} o(1),
$$

completing the proof.

\section{APPENDIX}

For the proof of Theorem 4.1, we first note that, as shown in BKR, the following holds as $x \rightarrow \infty$ :

$$
\begin{aligned}
\frac{\lambda^{\prime}(x)}{\lambda(x)^{2}} & \rightarrow 0, \\
\frac{\gamma^{\prime}(x)}{\sqrt{\lambda^{\prime}(x)} \gamma(x)} & \rightarrow 0 .
\end{aligned}
$$

In view of Proposition 3.2 in BKR, (10.2) need not hold for $\gamma$ itself but does for a tail equivalent version, with which $\gamma$ can be replaced without loss of generality. This implies

$$
\lambda \text { is flat for } \psi \text {. }
$$

Indeed, given $y$, for some $x^{*}$ between 0 and $x+y / \sqrt{\lambda^{\prime}(x)}$ we have

$$
\lambda\left(x+y / \sqrt{\lambda^{\prime}(x)}\right)=\lambda(x)+\frac{\lambda^{\prime}\left(x^{*}\right)}{\sqrt{\lambda^{\prime}(x)}} y=\lambda(x)+O\left(\sqrt{\lambda^{\prime}(x)}\right)=\lambda(x)(1+o(1)),
$$

where the $O(\cdot)$ estimate follows from a uniformity property of self-neglecting functions, and the $o(\cdot)$ estimate by (10.1). Using further (10.1) we see that $e=1 / \lambda$ is self-neglecting. 
Proof of Theorem 4.](i). Write $\bar{H}(x)=\gamma(x) \mathrm{e}^{-\psi(x)} / \lambda(x)$. Then

$$
\begin{aligned}
\bar{H}^{\prime}(x) & =\left[\gamma(x)+\frac{\gamma^{\prime}(x)}{\psi^{\prime}(x)}-\frac{\gamma(x) \psi^{\prime \prime}(x)}{\psi^{\prime}(x)^{2}}\right] \mathrm{e}^{-\psi(x)} \\
& =\gamma(x)\left[1+\frac{\gamma^{\prime}(x)}{\gamma(x) \psi^{\prime}(x)}-\frac{\psi^{\prime \prime}(x)}{\psi^{\prime}(x)^{2}}\right] \mathrm{e}^{-\psi(x)} .
\end{aligned}
$$

Here the last term in $[\cdot]$ goes to zero according to (10.1). This together with (10.2) also gives

$$
\frac{\gamma^{\prime}(x)}{\gamma(x) \psi^{\prime}(x)}=\frac{\gamma^{\prime}(x) \psi^{\prime \prime-1 / 2}}{\gamma(x)} \cdot \frac{\psi^{\prime \prime 1 / 2}}{\psi^{\prime}(x)}=o(1) \cdot o(1)=o(1) .
$$

Thus $\bar{H}^{\prime}(x) \sim f(x)$, which implies $\bar{H}(x) \sim \bar{F}(x)$.

We also have the following alternative proof for part (i).

Pro of of Theorem 4.](i). Using integration by parts yields

$$
\begin{aligned}
\int_{x}^{\infty} f(y) \mathrm{d} y & =\int_{0}^{\infty} \frac{\gamma(x+y)}{\psi^{\prime}(x+y)} \cdot \psi^{\prime}(x+y) \mathrm{e}^{-\psi(x+y)} \mathrm{d} y \\
& =\frac{\gamma(x)}{\psi^{\prime}(x)} \mathrm{e}^{-\psi(x)}-\int_{0}^{\infty} \frac{\mathrm{d}}{\mathrm{d} y}\left[\frac{\gamma(x+y)}{\psi^{\prime}(x+y)}\right] \cdot \mathrm{e}^{-\psi(x+y)} \mathrm{d} y .
\end{aligned}
$$

But by the same estimates as in the first version of the proof above, the first part of the integrand is $o(\gamma(x))$, so that the whole integral is $o(\bar{F}(x))$.

The following lemma is just a reformulation of part (ii) of the theorem, proved in BKR.

LEMMA 10.1. For any two pairs $\left(\gamma_{1}, \psi_{1}\right),\left(\gamma_{2}, \psi_{2}\right)$ satisfying the assumptions of Section $\square$, the integral

$$
\int_{-\infty}^{\infty} \gamma_{1}(z) \mathrm{e}^{-\psi_{1}(z)} \cdot \gamma_{2}(x-z) \mathrm{e}^{-\psi_{2}(x-z)} \mathrm{d} z
$$

has the asymptotics given by Theorem 1 .1] (ii).

Proof of The ore m 4. T(ii). This is a reformulation of Theorem 1.1 in BKR. Since, by (4.4), $q_{1}^{\prime}+q_{2}^{\prime}=1$, we have the claimed relation between $\lambda$ and $\lambda_{1}, \lambda_{2}$, namely

$$
\lambda(x)=\lambda_{1}\left(q_{1}(x)\right) q_{1}^{\prime}(x)+\lambda_{2}\left(q_{2}(x)\right) q_{2}^{\prime}(x)=\lambda_{1}\left(q_{1}\right)=\lambda_{2}\left(q_{2}\right) \text {, }
$$

establishing the proof. 
Proof of The ore m 4.](iii). We know that $\mathrm{e}^{-\psi_{i}(x)}, i=1,2$, is a von Mises function (see (8.1)), and thus $\mathrm{e}^{-\psi_{i}(x)} \in \operatorname{GMDA}\left(e_{i}\right), i=1,2$, with $e_{i}=$ $1 / \lambda_{i}$. Since further $e_{i}$ 's are self-neglecting and, by (10.1), $r_{i}(x)=\sqrt{\lambda_{i}(x)} / \lambda_{i}(x)$ $\rightarrow 0$ as $x \rightarrow \infty$, we have

$$
\lim _{x \rightarrow \infty} \frac{\gamma_{i}\left(x+e_{i}(x) y\right)}{\gamma_{i}(x)}=\lim _{x \rightarrow \infty} \frac{\gamma_{i}\left(x+y r_{i}(x) / \sqrt{\lambda_{i}(x)}\right)}{\gamma_{i}(x)}=1
$$

uniformly on bounded $y$-intervals. Hence $F_{i} \in \operatorname{GMDA}\left(e_{i}\right)$. Using Proposition 3.2 in BKR, we can find smooth $\gamma_{i}^{*}$ 's such that $\bar{H}_{i}(x)=\gamma_{i}^{*}(x) \mathrm{e}^{-\psi_{i}(x)} / \lambda_{i}(x)$ is asymptotically equivalent to $\bar{F}_{i}(x)$ as $x \rightarrow \infty$. Since also $H_{i} \in \operatorname{GMDA}\left(e_{i}\right)$ and $\lim _{x \rightarrow \infty} \lambda_{i}(x)=\infty$, for any $c>0$ we have

$$
\lim _{x \rightarrow \infty} \frac{\overline{H_{i}}(x+c)}{\bar{H}_{i}(x)}=0, \quad i=1,2 .
$$

Consequently, Corollary 1 in [11] yields $\overline{H_{1} * H_{2}}(x) \sim \overline{F_{1} * F_{2}}(x)$, and thus the claim follows from (ii).

By the above, we can find the asymptotics of $\overline{F_{1} * F_{2}}(x)$ assuming that $F_{i}$ 's have a density, so alternatively we get

$$
\overline{F_{1} * F_{2}}(x)=\int_{-\infty}^{\infty} \gamma_{1}(z) \mathrm{e}^{-\psi_{1}(x)} \cdot \frac{\gamma_{2}(x-z)}{\lambda_{2}(x-z)} \mathrm{e}^{-\psi_{2}(x-z)} \mathrm{d} z .
$$

But, by (10.3), $\gamma_{2} / \lambda_{2}$ is flat for $\psi_{2}$, so using Lemma 4.4 with $\gamma_{2}$ replaced by $\gamma_{2} / \lambda_{2}$, we can see that this integral asymptotically equals $\gamma(x) \mathrm{e}^{-\psi(x)} / \gamma_{2}\left(q_{2}(x)\right)$. But in view of (10.5) this is the same as $\gamma(x) \mathrm{e}^{-\psi(x)} / \lambda(x)$. This completes the proof.

Acknowledgments. EH is supported by SNSF Grant 200021-166274; PJL by an Australian Government Research Training Program Scholarship and an Australian Research Council Centre of Excellence for Mathematical \& Statistical Frontiers Scholarship.

\section{REFERENCES}

[1] A. A. Adler and G. Pakes, On relative stability and weighted laws of large numbers, Extremes 20 (2017), pp. 1-31.

[2] S. A smus sen, Conditional Monte Carlo for sums, with applications to insurance and finance, Ann. Actuar. Sci. (to appear in 2017); available at thiele.au.dk/publications.

[3] S. Asmussen and H. Albrecher, Ruin Probabilities, second edition, World Scientific, 2010.

[4] S. Asmussen and P. W. Glynn, Stochastic Simulation: Algorithms and Analysis, Springer, 2007.

[5] S. Asmussen and D. P. Kroese, Improved algorithms for rare event simulation with heavy tails, Adv. in Appl. Probab. 38 (2006), pp. 545-558. 
[6] A. A. Balkema and P. Embrechts, High Risk Scenarios and Extremes: A Geometric Approach, European Mathematical Society, Amsterdam 2007.

[7] A. A. Balkema, C. Klüppelberg, and S. I. Resnick, Densities with Gaussian tails, Proc. Lond. Math. Soc. 66 (1993), pp. 568-588.

[8] A. A. Balkema, C. Klüppelberg, and S. I. Resnick, Domains of attraction for exponential families, Stochastic Process. Appl. 107 (2003), pp. 83-103.

[9] O. E. Barndorff-Nielsen and C. Klüppelberg, A note on the tail accuracy of the univariate saddlepoint approximation, Ann. Fac. Sci. Toulouse Math. (6) 1 (1) (1992), pp. 5-14.

[10] D. B. H. Cline, Convolution tails, product tails and domains of attraction, Probab. Theory Related Fields 72 (1986), pp. 529-557.

[11] P. Embrechts, E. Hashorva, and T. Mikosch, Aggregation of log-linear risks, J. Appl. Probab. 51A (2014), pp. 203-212.

[12] P. Embrechts, C. Klüppelberg, and T. Mikosch, Modeling Extreme Events for Insurance and Finance, Springer, 1997.

[13] E. Hashorva and J. Li, Asymptotics for a discrete-time risk model with the emphasis on financial risk, Probab. Engrg. Inform. Sci. 28 (2014), pp. 573-588.

[14] J. L. Jen sen, Saddlepoint Approximations, Clarendon Press, Oxford 1995.

[15] S. I. Resnick, Extreme Values, Regular Variation, and Point Processes, Springer, 2008.

[16] H. Rootzén, A ratio limit theorem for the tails of weighted sums, Ann. Probab. 15 (1987), pp. $728-747$.

[17] T. Watanabe, Convolution equivalence and distributions of random sums, Probab. Theory Related Fields 142 (2008), pp. 367-397.

Søren Asmussen

Department of Mathematics

Aarhus University

Ny Munkegade

DK-8000 Aarhus C, Denmark

E-mail: asmus@imf.au.dk

Patrick J. Laub

University of Queensland \& Aarhus University

Department of Mathematics

University of Queensland, Brisbane

Queensland 4072, Australia

E-mail:p.laub@uq.edu.au
Enkelejd Hashorva

Department of Actuarial Science University of Lausanne

UNIL-Dorigny

1015 Lausanne, Switzerland E-mail: Enkelejd.Hashorva@unil.ch

Thomas Taimre Department of Mathematics University of Queensland, Brisbane Queensland 4072, Australia E-mail:t.taimre@uq.edu.au

Received on 15.2.2017;

revised version on 5.7.2017 